\title{
ALKAPTONUREA PRESENTING AS SPONTANEOUS RUPTURE OF TENDOACHILLIS
}

Ravikiran H. G1 , Prakash M², Pramod B. M³, Naveen N4, Mruthyunjaya5, Supreeth N6, Ganesh Ankam7, Vishnu Vardhan Reddy ${ }^{8}$

\section{HOW TO CITE THIS ARTICLE:}

Ravikiran H. G, Prakash M, Pramod B. M, Naveen N, Mruthyunjaya, Supreeth N, Ganesh Ankam, Vishnu Vardhan Reddy. "Alkaptonurea Presenting as Spontaneous Rupture of Tendoachillis". Journal of Evolution of Medical and Dental Sciences 2014; Vol. 3, Issue 71, December 18; Page: 15209-15213, DOI: 10.14260/jemds/2014/4046

INTRODUCTION: 'Ochronosis' literally meaning 'ochre or yellow appearance' (of the cartilage) was term used by Virchow to describe the musculoskeletal manifestation of alkaptonuria. Alkaptonuria is a rare autosomal recessive metabolic disorder. These patients have deficient homogentisic acid oxidase enzyme that is necessary for metabolism of homogentisic acid which in turn is produced from phenylalanine and tyrosine.[1]

The homogentisic acid when in excess gets polymerized and accumulates into connective tissues leading to dark pigmentation of tissues. The pigment is also has affinity towards hyaline cartilage of major peripheral joints and intervertebral discs.[2] Its deposition leads to early degenerative changes and arthritis. Along with discoloration of the skin, sclera and the pinnae, it may have systemic involvement of the genitourinary, cardiovascular and respiratory systems. ${ }^{[3]}$

Usually the disease progresses from simple alkaptonuria to alkaptonuric arthropathy by the fourth or fifth decade of life in $30 \%$ of patients. While degenerative changes of the spine and major joints have been frequently reported,[2-4] there are very few reports ${ }^{[5,6]}$ of spontaneous rupture of tendons caused by the deposition of pigmentat the osseo-tendinous junction.

We hereby report one such case where the patient presented with spontaneous rupture of tendon and on further investigations he was diagnosed as a case of alkaptonuria.

CASE: A 40 years old gentleman presented to us with pain the posterior aspect of his right leg and difficulty in walking which started suddenly while he was walking. There was no history of any injury. On examination he was found to have swelling over the posterior aspect of right ankle and inability to plantar flex the foot. Thompson "squeeze" test confirmed the discontinuity of the Achilles tendon (TA). Ultrasound scan and a radiograph were obtained and both of them confirmed the diagnosis of TA rupture.

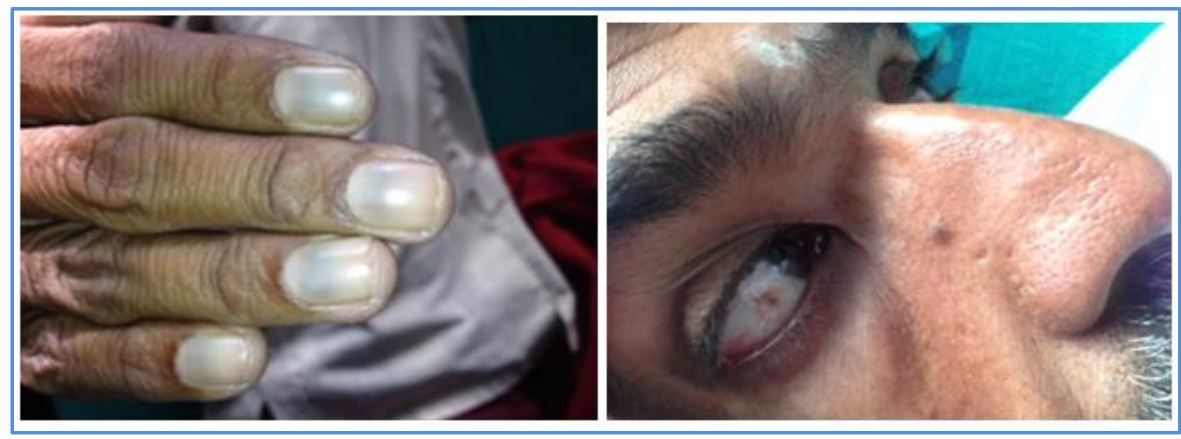

Fig. 1 \& 2: On examination: discoloration of the skin, nail, sclera and the pinnae 


\section{CASE REPORT}

The patient was posted for exploration and tendon repair. Exploration under anesthesia revealed which revealed complete tear of achilis tendon at insertion site. The tendon from the insertion site to the musculo-sketetal junction had blackish discoloration of the tendon. The tendon was soft and had rubbery consistency. Part of the tendon was excised and repair was achieved by $\mathrm{Z}$ lengthening of the remaining healthy tendon.

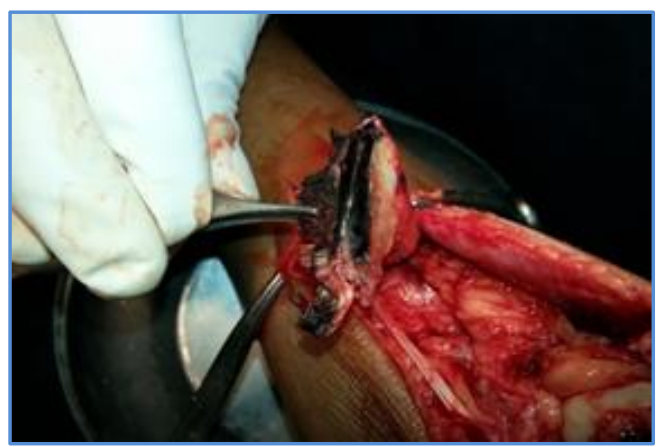

\section{Fig. 3: Blackish discoloration of the tendon}
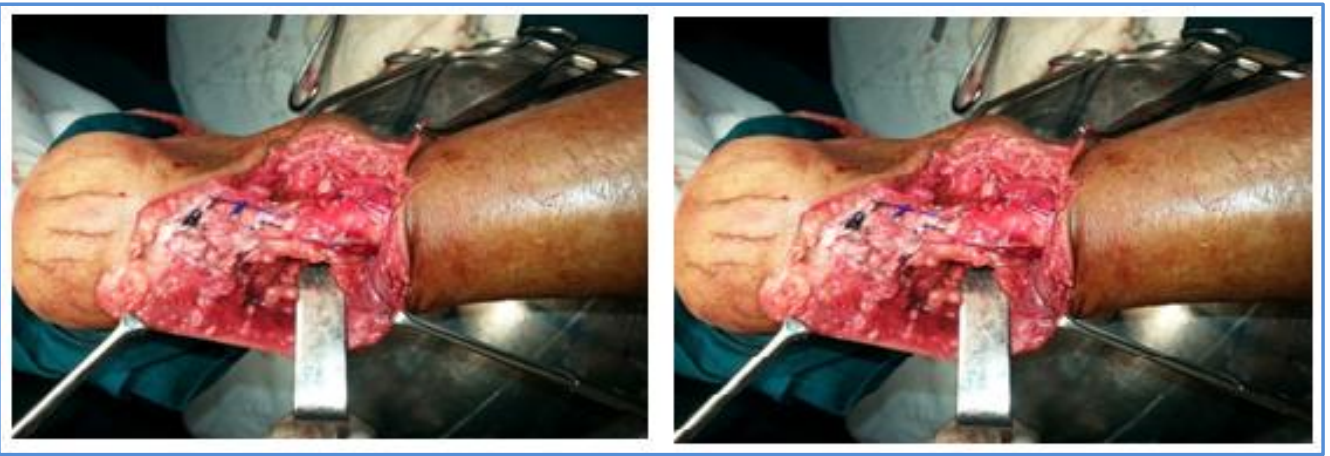

Fig. 4 \& 5: Part of the tendon was excised and repair done

The leg was immobilized in an above knee POP cast with ankle in around 30 degrees of equinous and was maintained for around four weeks. The wound inspection after four weeks revealed complete healing and the sutures were removed. Below knee cast was applied in equinous for another period of four weeks. At the end of eight weeks the wound had healed completely and the patient had no tenderness at the site of injury. Partial weight bearing was allowed at this time and patient was observed in outpatient on weekly basis. After 3 weeks after discontinuation of the plaster he was allowed to bear complete weight over the limb. Patient was followed up for nine months at monthly intervals.

The range of movements improved gradually and at nine months following the incident the he had 0-28 degrees of plantar flexion and 0-20 degrees of dorsiflexion. This was little more than 50\% of the normal side. He was able to manage his day to day activities and was able to walk comfortably. The wound site was healthy during this time and patient did not have any tenderness over the site of injury/ tendoachillis. 
We retrospectively collected detailed history and ordered a battery of investigations. Radiographs of his thoraco-lumbar spine revealed narrowing of disc spaces, calcification of disks and other degenerative changes. There was generalized osteopenia. The facet joints and sacroiliac joint though were un-involved. There was no involvement of sclera or ear cartilage. He did not have complains regarding peripheral joints.

ECHO showed-thickened mitral leaflets, mitral valve prolapsed, mild mitral stenosis with regurgitation. That ejection fraction was $60 \%$.

Histopathological examination of a resected sample of the tendon revealed yellow brown pigment (ochre) deposited in the chondrocytes and the tendon. All investigations of blood were with in normal limits. 24 hour urine sample turned dark colour and was negative for homogentisic acid. Other biochemical tests on urine confirmed the diagnosis of alkaptonurea. There was no family history though and the parents were not related.

DISCUSSION: Alkoptonuria (urine which turns dark on exposure to air), is a benign, rare inherited condition affection one in 250,000 to one million people worldwide. The disorder usually appears early in life and skeletal deformities can occur after third decade of life.

The homogentisic acid oxidase provides instruction for making an enzyme called homogentisic oxidase. This enzyme helps break down homogentisate in 50 maleyil acetoacetate. This results in derangement of metabolism of phenylalanine and tyrosine, which are building blocks of proteins. The mutation in HGD gene impairs the enzyme's role in this process. As a result homogentisate accumulates in the body. Upon contact with air, homogentisate is oxidised to form pigment like polymeric material responsible for the black color of urine. Although blood homogentisate levels are kept very low through rapid kidney clearance, over a period of time, homogentisate is deposited in cartilage throughout the body and is converted to the pigment like polymer through an enzyme-medicated reaction that occurs chiefly in collagenous tissues. As the polymer accumulates within cartilage, a process that takes many years, the normally transparent tissues become slate blue, an effect not seen until adulthood. The basic defect in alkaptonuria is deficient homogentisate 1, 2 dioxygenase activity. Nitisinone (A drug) inhibits 4-hydroxy phenylpyruvic acid dioxygenase as will be discussed in treatment options.

The earliest sign of the disorder is the tendency for diapers to stain black. In spine the symptoms in the lumbar and thoracic spine precede those in the cervical spine. Narrowing of the disc space was followed by disc calcification and fusion of the disc. Pigmentation of the sclera and ear cartilage occurred only after the age of thirty years and is extremely variable in severity; some have tendon related findings with thickened Achilles tendons and muscle tear after minimal trauma. Cardiac involvement includes aortic dilatation or cardiac-valve involvement i.e. aortic or mitral valve calcification or regurgitation on echocardiography. Urinary and plasma homogentisate levels can be quantitatively measured using spectrophotometric and chromatographic method.

The numerous reports on alkaptonuria are limited to degenerative manifestations in joints, but there are very few reports of the effects of the deposition of homogentisic acid in tendons leading to spontaneous rupture.[7,8] Homogentisic acid is widely deposited in connective tissue and such pigmentation must include tendons, which contain mainly type-I collagen. The accumulation of homogentisic acid inhibits collagen cross-linking leading to reduction of the structural integrity of collagen,3 thus increasing the likelihood of spontaneous rupture. ${ }^{[9]}$ The homogentisic acid oxidasw 
provides instruction for making an enzyme called homogentisic oxidase. This enzyme helps break down homogentisale in 50 maleyil acetoacetate. This results in derangement of metabolism of pherrylalanine and tyrosine, which are building blocks of proteins. The mutation in HGD gene impairs the enzyme is role in this process. As a result homogentisate accumulates in the body.

There is no specific treatment for alkaptonuricarthropathy; it includes physiotherapy, analgesia, and rest. Reduction of tyrosine and phenylalanine in the diet has been reported to reduce the excretion of homogentisate. It is not known whether dietary restriction from early life would avoid or minimise later complications, but such an approach is reasonable. Therapeutic method include mega dose of vitamin $\mathrm{C}$ (upto $1 \mathrm{gm} /$ day) for the degradation of homogentisic acid. Antiresorptive drugs such as alendronate and ibandronic acid are not proven to benefit in ochronotic spine with osteopenia. The aim of treatment is to halt the gradual destruction of cartilage, arthritis, lumbosacral ankylosis, limitation of motion, and deterioration of bone. Recently a new drug has been identified which inhibits 4-hydroxyphenylpyruvate dioxygenase. This is the enzyme that produces homogentisic acid. This drug is Nitisinone (NTBC) whose chemical name is 2- (2-nitro-4trifluoromethylbenzoyl) 1, 3 - cyclohexanedione and is sold under the brand name Orfadin. Nitisinone, at a dose of one $\mathrm{mg} / \mathrm{k}$ 'day is safe and effective in tyrosinemia type 1 but has not yet been approved for use in alkoptonuria.[10]

CONCLUSION: Ochronotic achillis tendon tear is a rare entity and can present with spontaneous Achilles tendon tear as an initial sign. In the presented case, excision of ochronotic tissue with lengthening of tendon by $\mathrm{Z}$ plasty done. In case of spontaneous Achilles tendon tear one has to keep in mind the unusual diagnose of Alkaptonuria.

\section{REFERENCES:}

1. Garrod AE, Oxon MD. The incidence of alkaptonuria: a study in chemical individuality. The Lancet 1902:161-20.

2. Dom K, Pittevils T. Ochronoticarthropathy, the black hip: case reportand review of the literature. Acta Orthop Belg 1997; 63: 122-5.

3. McColum DE, Odom GL. Alkaptonuria, ochronosis and low backpain: a case report. J Bone Joint Surg [Am] 1965; 47-A: 1389-92.

4. Borman P, Bodur H, Ciliz D. Ochronoticarthropathy. RheumatolInt2002; 21: 205-9.

5. Aydogdu S, Cullu E, Ozsoy MG, Sur H. Cementless total knee Arthroplastyin ochronoticarthropathy: a case report with four-year follow-up. J Arthroplasty 2000; 15: 539-43.

6. Carrier DA, Harris CM. Bilateral hip and bilateral knee arthroplastiesin a patient with ochronoticarthropathy. Orthop Rev 1990; 19: 1005-9.

7. Manoj Kumar RV, Rajasekaran S, Spontaneous tendon ruptures in alkaptonuria, JBJS, 2003,85-B (6); 883-6.

8. Shetty SS, Raj SNR ,Nirmal Babu P. , Mathias LJ ,BhatSP,Rai R. SpontaneousAcheles tendon rupture-A Case of Ochronosis. NUJHS, 2013, vol 3 (3); 113-5.

9. Emel E, Karagoz F, Aydin IH, Hacisalihoglu S, Seyithanoglu MH.Alkaptonuria with lumbar disc herniation: a report of two cases. Spine2000; 25: 2141-4.

10. Suzuki Y, Oda K, Yoshikawa Y, Maeda Y, Suzuki T. A novel the rapeutictrial of homogentisicaciduria in a murine model of alkaptonuria.J Hum Genet 1999; 44: 74-9. 


\section{AUTHORS:}

1. Ravikiran H. G.

2. Prakash M.

3. Pramod B. M.

4. Naveen N.

5. Mruthyunjaya

6. Supreeth N.

7. Ganesh Ankam

8. Vishnu Vardhan Reddy

\section{PARTICULARS OF CONTRIBUTORS:}

1. Assistant Professor, Department of Orthopaedics, JSS Medical College.

2. Senior Resident, Department of Orthopaedics, JSS Medical College.

3. Senior Resident, Department of Orthopaedics, JSS Medical College.

4. Senior Resident, Department of Orthopaedics, JSS Medical College.

5. Professor, Department of Orthopaedics, JSS Medical College.
6. Senior Resident, Department of Orthopaedics, JSS Medical College.

7. Post Graduate, Department of Orthopaedics, JSS Medical College.

8. Post Graduate, Department of Orthopaedics, JSS Medical College.

\section{NAME ADDRESS EMAIL ID OF THE} CORRESPONDING AUTHOR:

Dr. Ravikiran H. G, \# 884, 4th Main,

S. S. Nagar, Mysore-570015.

Email: ravikiranhg@gmail.com

Date of Submission: 05/12/2014. Date of Peer Review: 06/12/2014. Date of Acceptance: 13/12/2014. Date of Publishing: 18/12/2014. 